\title{
LA DISTRIBUCIÓN GEOGRÁFICA E INTERÉS ETNOBOTÁNICO DE PETALOSTELMA ROBERTII (APOCYNACEAE) EN EL GRAN CHACO
}

\author{
PASTOR ARENAS ${ }^{1} \&$ SERGIO A. CÁCERES MORAL ${ }^{2}$
}

\begin{abstract}
Summary: Arenas, P. \& S. A. Cáceres Moral. 2013. Geographical distribution of Petalostelma robertii (Apocynaceae) in the Gran Chaco and its interest for ethnobotany. Bonplandia 22(2): 181-190.

This paper discusses the role played by Petalostelma robertii (S. Moore) Liede \& Meve (Apocynaceae) in the ethnobotany of two ethnic groups from the Argentine semi-arid Chaco: the Wichí and the Toba-Pilagá peoples, who use it as a love spell. It provides data on the morphology, ecology and geographical distribution of the plant, as well as references on its significance in the social and spiritual lives of these ethnic groups. Its presence in Argentina is mentioned for the first time, and the morphological similarities and discrepancies in the collections made in different localities in the region are discussed. The provisional nature of the identity given to the plant is categorically stated. Its ethnobotanical interest and the need to assign a scientific name to the plant, at least an approximate one, are discussed. On the taxonomic and floral levels, the conclusion is that a detailed study of the exsiccata available is required in order to clarify the identity of a group of plants related to $P$. robertii.
\end{abstract}

Key words: Ethnobotany, love magic, Wichí indians, Toba-Pilagá indians.

Resumen: Arenas, P. \& S. A. Cáceres Moral. 2013. La distribución geográfica e interés etnobotánico de Petalostelma robertii (Apocynaceae) en el Gran Chaco. Bonplandia 22(2): 181190.

Se da a conocer el papel que desempeña Petalostelma robertii (S. Moore) Liede \& Meve (Apocynaceae) en la etnobotánica de dos grupos étnicos del Chaco semiárido argentino: los Wichí y los Toba-Pilagá, quienes la aplican para la magia de amor. Se aportan datos sobre morfología, ecología y distribución geográfica de la planta, así como referencias sobre su interés en la vida social y espiritual de las etnias involucradas. Se menciona por primera vez su presencia en Argentina y se discuten las concordancias y discrepancias morfológicas existentes en colecciones provenientes de distintas localidades de la región. Se deja asentado el carácter provisional de la identidad adjudicada. Se discute el interés etnobotánico de la planta y la necesidad de dotarle de un nombre científico aunque fuere aproximado. Se concluye, en el plano taxonómico y florístico, en la necesidad de un estudio minucioso de la exsiccata disponible a fin de esclarecer la identidad de un grupo de entidades relacionadas con $P$. robertii.

Palabras clave: Etnobotánica, magia de amor, indígenas Wichí, indígenas Toba-Pilagá.

\footnotetext{
${ }^{1}$ Centro de Estudios Farmacológicos y Botánicos, CEFyBO-CONICET, Facultad de Medicina, Universidad de Buenos Aires, Paraguay 2155, Piso 16 (1121), Buenos Aires, Argentina. pastorarenas@yahoo.com.ar

2 Instituto de Botánica del Nordeste, IBONE-CONICET. Facultad de Ciencias Agrarias, Universidad Nacional del Nordeste, FCA-UNNE. C. C. 209, 3400 Corrientes, Argentina. qcaceress@gmail.com
} 


\section{Introducción}

Durante el desarrollo de trabajos etnobotánicos entre los Wichí y Toba-Pilagá (= toba-ñachilamole'ek) del Chaco semiárido argentino recolectamos una planta que tiene gravitación en la vida social y espiritual de estos dos pueblos nativos. Consideramos que esta planta corresponde a Petalostelma robertii, una trepadora propia del Gran Chaco. Fue descripta como novedad para la ciencia con el nombre Vincetoxicum robertii $\mathrm{S}$. Moore (Moore, 1904: 104), sobre la base de un espécimen proveniente de Corumbá, un puerto fronterizo brasileño de Mato Grosso do Sul, en la ribera izquierda del río Paraguay. Otras colecciones posteriores mostraron una distribución localizada en la región del Alto Paraguay y Mato Grosso do Sul, todas ellas de principios del siglo XX (Liede \& Meve, 2001: 176, fig. 1). Posteriormente, en las décadas siguientes, fueron incorporándose en herbarios de Argentina y Paraguay nuevas colectas que pueden adscribirse a esta especie. Estos pliegos, hay que hacerlo notar, son muy contados y comprenden sitios de recolección muy dispersos y apartados (Fig. 1). Este material permanecía guardado en los herbarios como indeterminados, como pertenecientes a otros géneros o sin poder lograrse una identificación convincente de los mismos.

Los ejemplares reunidos por P. Arenas en la década de 1980, en el Chaco semiárido de las provincias argentinas de Salta y Formosa, sólo se podía vincular mediante la bibliografía local asequible con Petalostelma sarcostemma (Lillo) Liede \& Meve, única especie del género que vive en el noroeste argentino. Esta especie, resulta parecida en primeras instancias, pero una observación detallada mostró que el material en estudio se trata de una entidad diferente, sobre todo, si se coteja con la exsiccata de Cynanchum sarcostemma, nombre con el que la citaron los monógrafos de la familia (Lillo, 1918/19; Malme, 1933; Meyer, 1944, 1977). Esta situación hizo necesario que se indagara con mayor profundidad la identidad del material que era motivo de nuestro estudio. Además de las diferencias morfológicas, una de las primeras observaciones llamativas que surgía era el área de distribución de $P$. sarcostemma, que parecía circunscripto o limitado al Chaco serrano. En efecto, su presencia fue registrada en Salta y Tucumán, un área de distribución que se mencionó reiteradamente con el paso de los años, con una exsiccata que en su mayor parte no se acrecentó (Hechem \& Ezcurra, 2006; Liede \& Meve, 2001; Malme, 1933; Meyer, 1944, 1977). Recientemente, Liede \& Meve (2001) revisaron la posición sistemática de esta especie así como de otras relacionadas y dieron a conocer el estatus actual de las mismas. El concepto de estos autores es el que empleamos en este trabajo. Así pudimos relacionar las plantas investigadas con $P$. robertii.

La finalidad de esta contribución es ampliar el área de distribución de $P$. robertii en la zona de planicies secas del Gran Chaco, que incluye a Argentina, Paraguay y Bolivia, que se suma a la zona limítrofe del Mato Grosso do Sul brasileño, que ya era conocida. Al mismo tiempo, se brindan sucintos datos florísticos, morfológicos y ecológicos que permiten acrecentar el conocimiento sobre la planta en cuestión. Estos datos se centran en los ejemplares que fueron observados in situ.

Asimismo, deseamos resaltar su importancia en la etnobotánica de indígenas de la región. En efecto, la planta trasciende su materialidad como vegetal y tiene un papel y significado relevante en determinados contextos de la vida social de pueblos originarios de la región, según se detallará más abajo.

\section{Material y Método}

La planta se obtuvo durante trabajos de campo en el marco de investigaciones etnobotánicas entre indígenas del Gran Chaco. Se aplicaron las metodologías propias de la etnociencia, que en este caso fueron de carácter cualitativo (Arenas \& Martínez, 2012). Para abordar este tema se tuvo en consideración las instancias propias de este enfoque: a) la visión y percepción del actor (el trabajo etnográfico) y b) la aplicación del enfoque científico (el trabajo de gabinete y laboratorio). En la primera etapa se realizaron entrevistas con informantes calificados, observaciones in situ, conversaciones ocasionales, entre otros mecanismos de recolección de datos. Se 


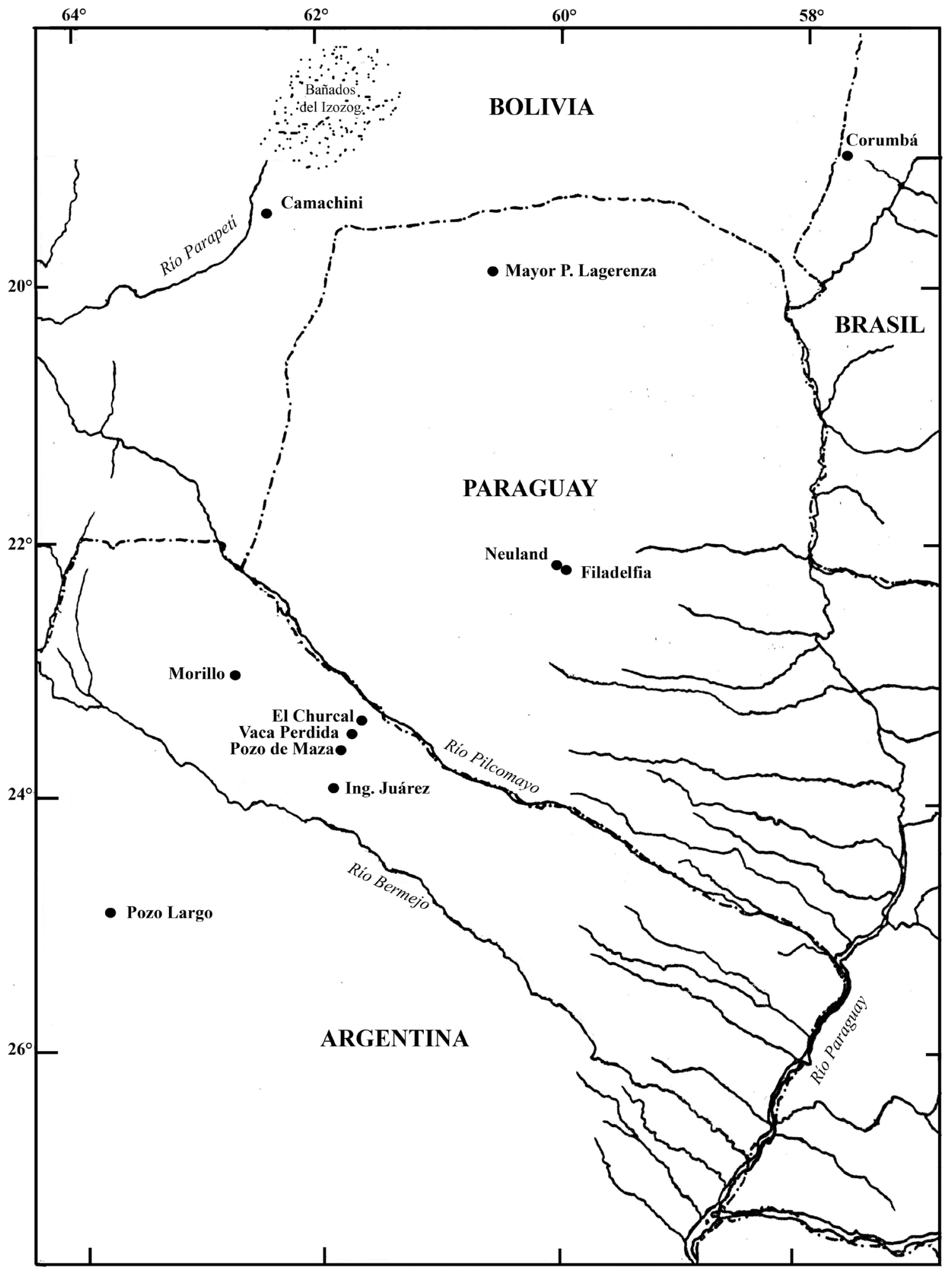

Fig.1. Gran Chaco. Sitios de recolección de Petalostelma robertii. 
aplicó una encuesta ad hoc diseñada para estos trabajos (Arenas, 1995). El material vegetal de referencia se obtuvo durante recorridas en el campo en compañía de los informantes; las muestras reunidas se prepararon para su posterior identificación en el laboratorio. Se tomaron notas, grabaciones y fotografías que documentan el material de herbario y las referencias orales.

La identificación del material de herbario se realizó según las pautas habituales: estudio detallado del espécimen, cotejo con la bibliografía específica y especializada, consulta con especialistas y la revisión de material de herbario comparativo. El material de herbario revisado se divide en dos grupos: a) material estudiado, que comprende de manera específica los ejemplares que forman parte del estudio etnobotánico y constituye el motivo central de esta nota, b) material comparativo estudiado, corresponde a los ejemplares utilizados para confrontar, corroborar y discutir la identidad de la especie.

Material estudiado: ARGENTINA. Formosa: Dep. Bermejo, Pozo de Maza, 31-I-1989, Arenas 3394 (BA, CTES); El Churcal, 28-XI-1985, Arenas 3084 (BA, CTES); Vaca Perdida, 20-III-1986, Arenas 3235 (BA, CTES). Dep. Matacos, 6 km en bosque alto, alrededores de Ing. G. N. Juárez, 24-I-1987, Arenas 3245 (BA, CTES); Salta: Dep. Rivadavia, Morillo, alrededores de la Toldería, 1-I1984, Arenas 2559 (BA, CTES); Morillo, 26-VI1984, Arenas 2754 (BA, CTES).

Material comparativo estudiado: ARGENTINA. Salta: Dep. Anta, Pozo Largo, 15-III-1986, Saravia Toledo 1188 (CTES). PARAGUAY. Boquerón: Col. Fernhein, Filadelfia, VII-2000, August y Ulmke 61: Col. Neuland, 24-VII-1992, Degen y Mereles 2695 (FCQ). Chaco: Mayor P. Lagerenza, $20^{\circ} \mathrm{S}$ $60^{\circ} 45^{\prime} \mathrm{W}, 16-\mathrm{IV}-1978$, Schinini y Bordas 15109 (CTES).

P. aff. robertii. BOLIVIA. Santa Cruz. Camachini, cerca del río Parapití, III-1935, Rojas 7223 (CTES, LIL).

P. sarcostemma. ARGENTINA. Salta. Dep. Capital, Cerro de San Bernardo, 1200 m n.s.m., 18III-1927, Schreiter 5225 (LIL). Dep. Rosario de la
Frontera, Rosario de la Frontera, 900 m n.s.m., 26III-1905, Lillo 4410 (LIL). Tucumán. Dep. Capital, Barranca Colorada, 700 m n.s.m., V-1920, Venturi 809 (BA, LIL); Barranca Colorada, 500 m n.s.m., 30-X-1920, Schreiter 1378 (LIL); Barranca de la Toma (río Salí), 450 m n.s.m., 1-III-1921, Schreiter 1677 (LIL), Barranca de la Toma (río Salí), 450 m n.s.m., 1-III-1921, Schreiter s.n. (LIL 30529): Barranca de la Toma (Dique río Salí), 500 m n.s.m., 17-IV-1921, Schreiter 1637 (LIL 30948). Dep. Trancas, Vipos, 900 m n.s.m., 30-I-1922, Venturi 1677 (LIL); Vipos, 786 m n.s.m., 3-XII-1922, Schreiter 2465 (LIL).

\section{Resultados}

Petalostelma robertii fue señalada como estrechamente afín a $P$. sarcostemma, única especie del género citada para el noroeste de Argentina. Se diferencia de ella por el tamaño menor de sus hojas y flores, comparten la inserción lateral de las caudículas en los polinios (Malme, 1933: 28; Liede \& Meve, 2001:176), e inflorescencias paucifloras cortamente pedunculadas, con delicadas flores (Liede \& Meve, 2001:176).

Las descripciones e ilustraciones de $P$. sarcostemma, son un tanto contradictorias según los distintos autores. Estas discrepancias en las apreciaciones sobre la morfología floral se refieren principalmente al indumento de la corola, las características de la corona, y el lugar de inserción de las caudículas en el retináculo. Lillo (1919: 424), en la descripción original de Cynanchum sarcostemma, no hace referencia a la presencia de pelos largos en la corola; Malme (1928: 28) los menciona pero no los ilustra, en tanto Meyer (1944: 152, tab. 71) los consigna en la descripción como pelos lanuginosos, pero curiosamente omite el indumento mencionado en la ilustración. Liede \& Meve (2001: 176, fig. 6) señalan la presencia de tricomas bastante largos, distribuidos regularmente sobre la corola.

Con respecto a las características de la corona, para Lillo (1919: 424) y Meyer (1944: 152, tab. 71) los lóbulos son carnosos, enteros, redondeados, observándose en la ilustración que se disponen paralelos y adosados al ginostegio. Para Malme (1928: 28) es 5-fida 

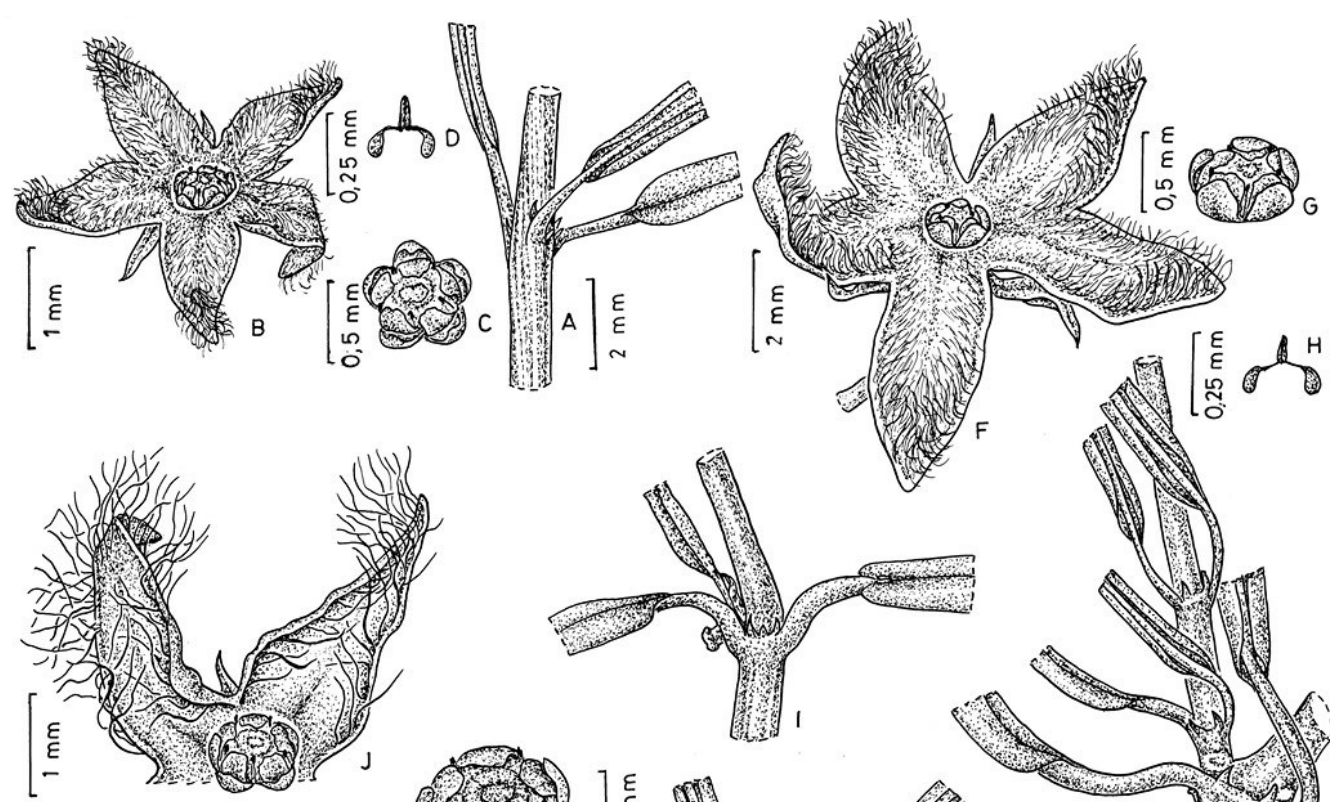

I唇 alor
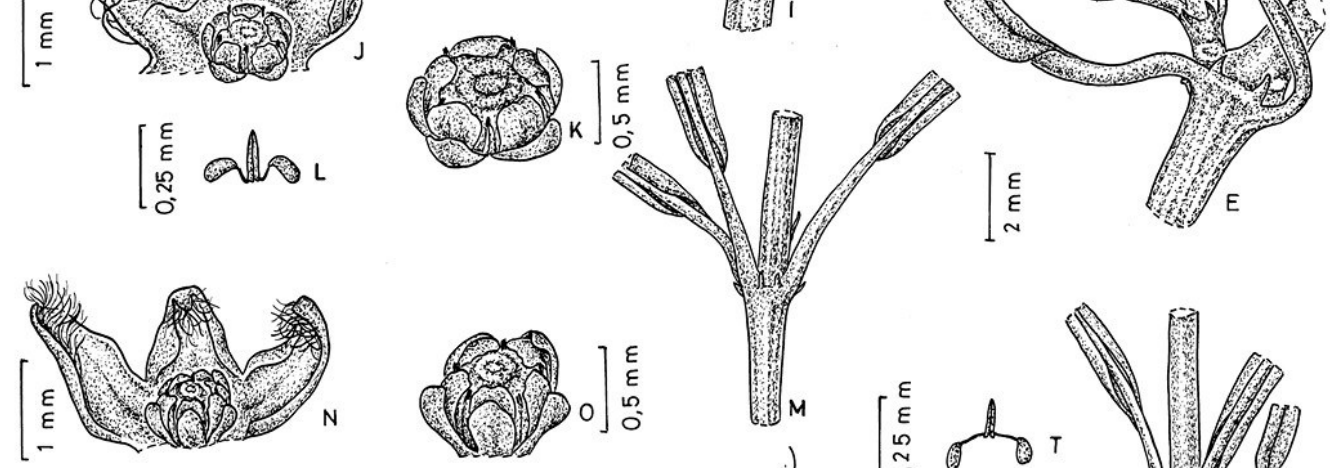

点

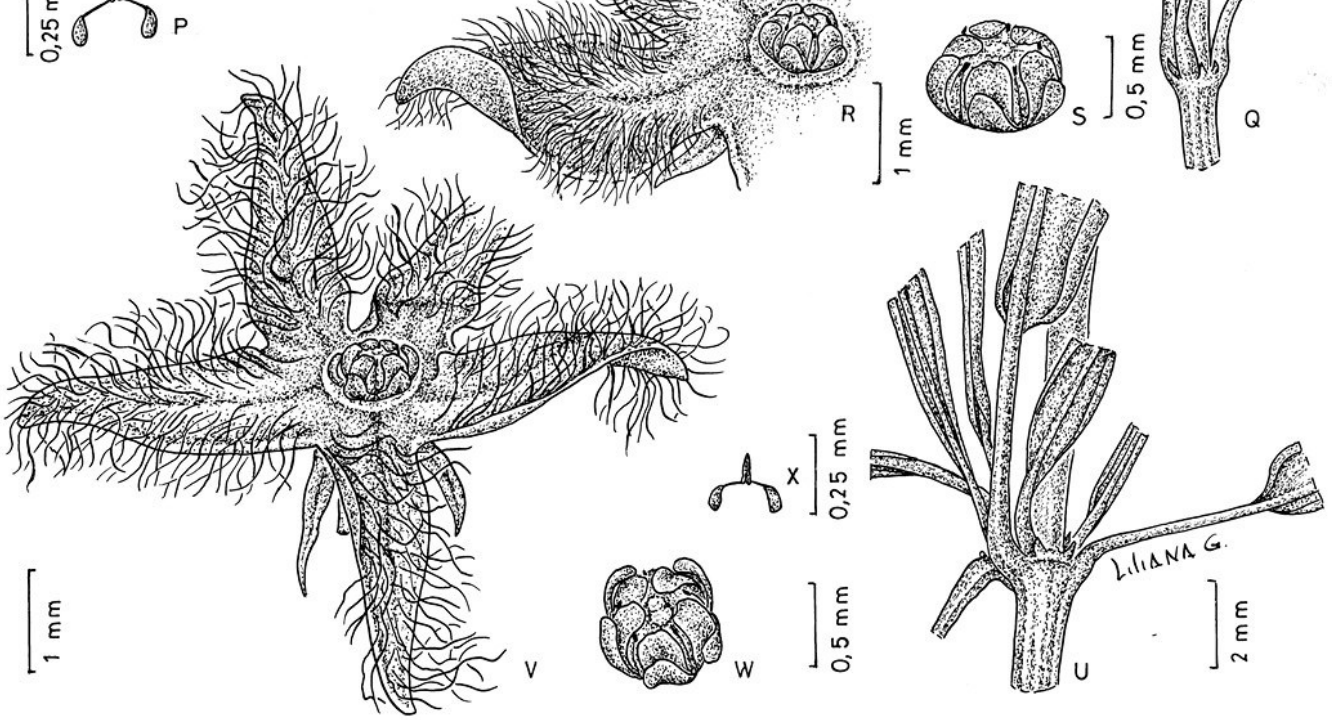

Fig.2. Petalostelma robertii. A: Hojas. B: Flor. C: Ginostegio. D: Polinario (Arenas 3084). E: Hojas. F: Flor. G: Ginostegio. H: Polinario (August y Ulmke 61). I: Hojas. J: Flor. K: Ginostegio. L: Polinario (Saravia Toledo 1188). M: Hojas. N: Flor. O: Ginostegio. P: Polinario (Schinini 15109). Q: Hojas. R: Flor. S: Ginostegio. T: Polinario (Arenas 2754). U: Hojas. V: Flor. W: Ginostegio. X: Polinario (Arenas 3394). Dibujó Liliana Gómez. 
con lóbulos semi-orbiculares, pero no ilustra; en tanto Liede \& Meve (2001: 176, fig. 6) señalan lóbulos libres, carnosos, y en la ilustración se aprecia claramente que son patentes, dispuestos casi perpendicularmente con respecto al ginostegio.

Por último, observando las ilustraciones del polinario de $P$. sarcostemma, para Meyer las caudículas se insertan en la base del retináculo, en tanto que para Malme (1928: 27), Liede \& Meve (2001: 176) las mismas se insertan apenas por debajo de la parte media del retináculo.

Durante el estudio de los ejemplares del Chaco argentino, hallamos en los distintos ejemplares analizados caracteres morfológicos tanto de $P$. sarcostemma como de $P$. robertii, los cuales se pueden apreciar en la Fig. 2. El tamaño de las flores es variable como también el indumento de la corola. Los pelos largos se asemejan a los de las flores de P. sarcostemma, tal como ilustran Liede \& Meve (2001: 178, fig. 6 B), y se distribuyen uniformemente, o pueden localizarse principalmente en los bordes o en el ápice de los pétalos. Ninguno de los ejemplares estudiados presenta la corola cubierta sólo de pelos cortos propios de $P$. robertii (Liede \& Meve, 2001: 177, fig. 5 C). En cuanto a la corona, está dividida en lóbulos más o menos patentes similares a los de $P$. sarcostemma. Con respecto al polinario, nuestros ejemplares también muestran algunas variaciones en el tamaño del retináculo, pero en ninguno las caudículas se insertan en la parte media del retináculo.

Los caracteres morfológicos que nos inclinan a identificar los ejemplares estudiados como $P$. robertii son las caudículas insertas en la base del retináculo y principalmente las hojas angostas, revolutas, graminiformes insertas en braquiblastos. Este rasgo constituye el carácter distintivo de esta especie, según resaltan tanto Moore (1904: 104) como Liede \& Meve (2001: 176). Los últimos autores son aún más enfáticos en diferenciarla de las demás especies de Metastelminae de Sudamérica por ser la única cuyas hojas se disponen de esta manera.

Seguidamente reproducimos algunas de las observaciones realizadas in situ con el material estudiado (Fig. 3).
Petalostelma robertii es una enredadera grácil, latescente, con flores y frutos de coloración intensa y llamativa. Posee un órgano subterráneo, con un eje central breve del cual emergen raíces angostas y profundas. Este órgano, que los nativos llaman "raíces", cuando se raspa desprende un aroma agradable, siendo ésta la porción empleada por ellos. Las inflorescencias son péndulas; las flores tienen la corola rotada de un color granateviolado oscuro y presenta una apariencia aterciopelada debido a los pelos largos y sedosos que la cubren. Los frutos son de color verdoso claro, con manchas ferrugíneas. Las hojas angostas presentan la haz de color verde oscuro, amarronado, en tanto que el envés es verde claro. Omitimos otros detalles de su morfología ya que las descripciones existentes son suficientemente ilustrativas (Liede \& Meve, 2001; Malme, 1927: 7-8; Moore, 1904: 104).

\section{Ecología y florística}

El material estudiado se colectó en la llanura seca chaqueña; habita en el bosque primario, en terrenos elevados donde se destacan las especies características de la región: "palosanto" (Bulnesia sarmientoi Lorentz ex Griseb.), "quebracho blanco" (Aspidosperma quebracho-blanco Schltdl.), "quebracho" (Schinopsis lorentzii (Griseb.) Eng1.), "algarrobos" (Prosopis spp.), "guayacán" (Caesalpinia paraguariensis (D. Parodi) Burkart), "palo mataco" (Prosopis kuntzei Harms), entre otros. También se la puede encontrar en montes densos de menor altura, con poca accesibilidad, conformados por "escayante" (Mimozyganthus carinatus (Griseb.)Burkart), "teatín”(Acacia fuscatispina Griseb.), "duraznillo" (Salta triflora (Griseb.) Adr. Sánchez), "garabato" (Acacia praecox Griseb.), "brea" (Cercidium praecox (Ruiz \& Pav. ex Hook. \& Arn.) Harms), entre otros. Su fenología es curiosa; se la colectó en floración y fructificación tanto en primavera-verano (noviembre a marzo) como en pleno invierno (junio).

Otro de los puntos de interés de esta contribución se refiere a la ampliación del área de distribución de $P$. robertii, que incluye el Chaco semiárido de las provincias argentinas 


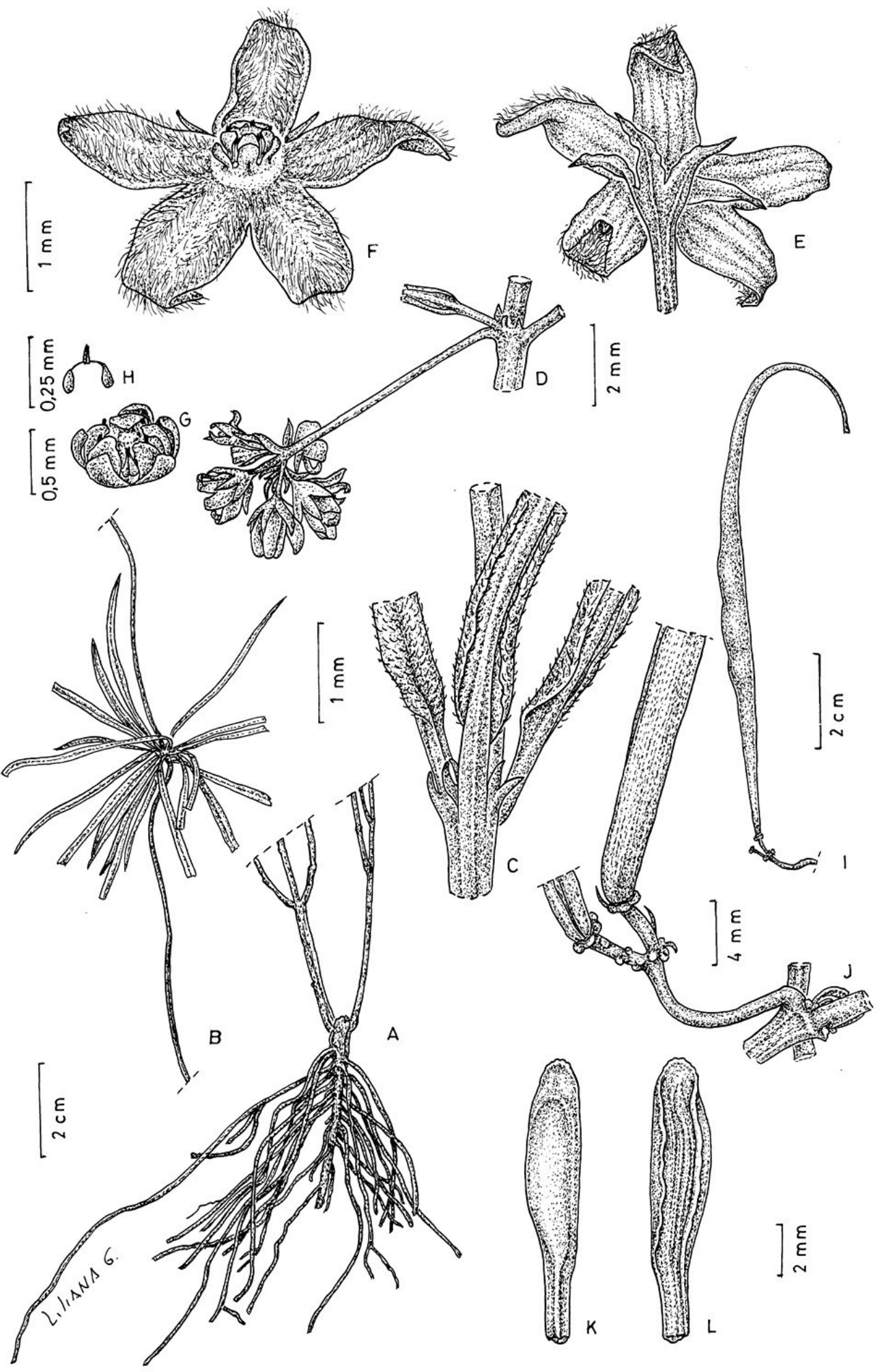

Fig. 3. Petalostelma robertii A: Raíz (Arenas 3084). B-C: Hojas (Arenas 2559) . D: Inflorescencia. E-F: Flor. G: Corona y ginostegio. H: Polinario. I-J: Fruto. K-L: Semilla (Arenas 3084). Dibujó Liliana Gómez. 
de Salta y Formosa, por lo cual este dato constituye una nueva cita para Argentina. Su presencia en Paraguay ya era conocida en áreas relativamente cercanas a la localidad tipo (Corumbá) del Alto Paraguay y el Mato Grosso do Sul brasileño (Moore, 1904: 124, Malme, 1927: 7; Liede \& Meve, 2001: 176). Hay que señalar, asimismo, su presencia en el interior del Chaco semiárido del Paraguay, dato que fue publicado por Ulmke \& August (2004: 120-121) en una guía de plantas del Chaco seco, en donde constan tres fotos en color que resultan muy ilustrativas. El material que documenta dicho trabajo fue citado como Cynanchum sarcostemma, el cual fue estudiado por nosotros y es mencionado en la exsiccata de esta contribución.

Otro material interesante a ser estudiado, que consideramos como afín a $P$. robertii pertenece al botánico paraguayo Teodoro Rojas, quien lo coleccionó en el curso de la Guerra del Chaco (1932-1935), formando parte de la retaguardia del ejército y partícipe de la conquista del río Parapiti (Schinini, 2005: 106, 134-135). Este material de Rojas presenta muchas similitudes con $P$. robertii, especialmente en las hojas graminiformes pseudoverticiladas, la corona y polinario. Sin embargo, posee flores tubulosas, lo cual nos impide ubicarlo con certeza bajo este nombre. En cuanto a la localidad y circunstancias de la colecta realizada por T. Rojas es necesario efectuar algunas aclaraciones. El sitio pertenecía a una zona por aquel entonces en litigio entre Paraguay y Bolivia. Cuando concluyó el conflicto, en el Tratado de Paz entre ambos países (firmado en Buenos Aires, 21 de julio de 1938) se asignó dicho territorio a Bolivia. Rojas consigna en su etiqueta la localidad "Camachini, cerca del río Parapití', que fue imposible localizar. No obstante los datos históricos indican que en la fecha mencionada, las tropas paraguayas cruzaban el río Parapití y el 1-III-1935 accedían a un poblado llamado Tamachindi, que quizá sea el nombre del lugar, y fuera mal transcripto por Rojas (Sienra Zavala, 2010: 204-218; Joy, 1992: 149).

\section{Etnobotánica}

El interés etnobotánico de esta planta radica en que los integrantes de las etnias Toba-Pilagá del oeste de la provincia de Formosa y Wichís del Chaco salteño aplican las raíces de esta planta como amuleto para lograr la atracción y el amor de una persona deseada. Los colores y la belleza de la planta, así como su escasez y muy ocasional hallazgo, son datos que influyen para que la planta sea cotizada y muy valorada. Su hallazgo no constituye una mera casualidad y de hecho la planta y su empleo se relacionan con vínculos que posee el vegetal con entes y poderes sobrenaturales. Cuentan los relatos, que encontrar esta planta, a veces por aparente azar, en realidad se debe a una dádiva intencional que proviene de un ente sobrenatural que -por distintas razones- le provee como una suerte de obsequio a quien la encuentra, lo cual considera el afortunado como una suerte de signo o revelación. Esta circunstancia -de excepcional hallazgo- la dota de "encanto" y de poderes que le sirve al usuario para lograr el fin deseado. La parte usada es la "raíz", que se deseca y se mantiene oculta y en secreto dentro de una bolsita o envoltorio. La persona que realiza el hechizo lleva este material consigo entre la ropa, colgado como un collar, en bolsillos, etc., y se considera que mediante su "olor" o efluvios penetra en el espíritu de la persona requerida. Esta raíz suele ir acompañada de otras plantas aromáticas también aplicadas en magia de amor, y se le adicionan fragancias (jabón de olor, talco, colonias, etc.) que potencian el perfume propio de la raíz.

Si bien estas cualidades o categorías de uso que se atribuyen a las plantas pueden resultar trivialidades o anécdotas risueñas a los ojos de la cultura occidental, en los sitios donde tienen su escenario son de notable importancia. Situémonos, para comprender su alcance, en lo que representa la cuestión sentimental y amorosa en nuestra sociedad. Las mismas pulsiones, que son propias de la humanidad, se dan entre los nativos en cuestiones de amor: el deseo, la frustración, el rechazo, las dudas, las indecisiones, es decir, los males de amores. Pasión, dolor, angustia, regocijo, entre otras situaciones, se dan lugar por carencia y por el afán del logro del cometido amoroso, y son estas plantas un motivo de gran interés personal en distintos momentos de la vida de la gente. Este tema, dada sus características 
y particularidades en el plano de la cultura forman parte de sendos trabajos, dedicados tanto a los toba-pilagá como a los Wichí, que se darán a conocer próximamente, lo cual nos exime de extendernos en este trabajo en el tema específicamente etnográfico.

\section{Discusión y Conclusiones}

El interés por contar con una determinación fiable, o la más aproximada posible, no constituye una actitud pretenciosa sino la de situar el dato etnobotánico de una manera cercana a la especie en concreto y no reducirla a la vaguedad de un nombre a nivel de género. Los especímenes que sirven para documentar este trabajo reúnen características morfológicas que permite identificarlos como $P$. robertii. Sin embargo, hay que resaltar que el material del Chaco argentino estudiado muestra una variación dentro de las propias colecciones, lo cual hace difícil determinarlos con total certeza; tampoco contribuye a esclarecer el problema las diferencias observables en la literatura así como en las ilustraciones que éstas presentan, según señalamos en los resultados. A estas dificultades se suman la gran dispersión de estas plantas en la geografía chaqueña, la escasez de colecciones, así como la existencia de ejemplares no estudiados por especialistas en los herbarios locales. Por tales razones consideramos que el caso ameritaría una investigación más detallada para establecer correctamente las pertenencias de las especies. Consideramos que la nomenclatura aceptada para estas plantas en este trabajo constituye una solución momentánea hasta que tengamos nuevas revisiones del género.

Desconocemos la naturaleza del órgano subterráneo empleado; podría tratarse de una estructura caulinar adaptada para los largos períodos de sequía que debe soportar la planta. Sin embargo, no realizamos estudios morfológicos que puedan verificar si se trata de un xilopodio o de un rizoma vertical breve, tarea que debería ser encarada a futuro.

Uno de los elementos de juicio que inducen a mayor duda es la enorme dispersión de los sitios de colecta, que se sitúan en enclaves a cientos y a miles de kilómetros de distancia entre uno y otro individuo, quedando en medio amplísimos espacios vacíos (Fig. 1). Una revisión de un mayor número de muestras puede explicar si las variaciones morfológicas responden a las fluctuaciones propias dentro de una entidad o si se trata de taxones diferentes.

\section{Agradecimientos}

P. Arenas agradece a integrantes de las etnias Toba-Pilagá del oeste de la provincia de Formosa y Wichís de la localidad de Morillo (Salta), del Chaco argentino, por sus datos y aportes para dar a conocer este tema propio de sus culturas. Al profesor Gilberto Morillo, especialista en asclepiadáceas, de la Universidad de Mérida (Venezuela), que nos prestó su ayuda para identificar numerosas especies chaqueñas. Sobre el material estudiado en este caso nos dio consejos, orientaciones y nos marcó sus dudas.

A las autoridades y curadores de los herbarios mencionados que permitieron el acceso y consulta de las colecciones, así como por facilitarnos el préstamo de algunos ejemplares. A Liliana Gómez por las ilustraciones que acompañan este texto. A María Eugenia Suárez por su ayuda en la preparación del mapa, y al Ing. Agr. Antonio Krapovickas por sus útiles indicaciones. Nuestra gratitud especial a los profesores Sigrid Liede y Jorge Fontella Pereira, que aportaron informaciones y enriquecieron el trabajo con sus opiniones.

\section{Bibliografía}

ARENAS, P. 1995. Encuesta general de etnobotánica aplicada a indígenas del Gran Chaco. Hacia una carta étnica del Gran Chaco VI. Centro del Hombre Antiguo Chaqueño (CHACO), Las Lomitas (Formosa, Argentina), pp. 161-178.

— \& G. J. MARTÍNEZ. 2012. Estudio etnobotánico en regiones áridas y semiáridas de Argentina y zonas limítrofes. Experiencias y reflexiones metodológicas de un grupo de investigación. En P. Arenas (ed.), Etnobotánica en regiones áridas y semiáridas del Cono Sur de Sudamérica, pp. 11-43. Edición del CEFYBO-CONICET, Buenos Aires.

HECHEM, V. \& C. EZCURRA. 2006. Asclepiadaceae. Aport. Bot. Salta, Ser. Fl. 7 (13): 1-62. 
JOY, J. C.1992. Los fortines de la guerra: Toponimia chaqueña. Ed. Estudio Gráfico, Asunción. 159 pp.

LIEDE, S. \& U. MEVE. 2001. Taxonomic changes in American Metastelminae (ApocynaceaeAsclepiadoideae). Novon 11: 171-182.

LILLO, M. 1918/19. Las asclepiadáceas argentinas. Physis 4: 410-437.

MALME, G. O. A. 1927. Asclepiadaceae mattogrossenses. Ark. Bot. 21A (12): 1-27, Tab. I.

- 1933. Asclepiadaceae argentinae. Ark. Bot. 26 A, 4: 1-45.

MEYER, T. 1944. Asclepiadaceae. En H. R. Descole (ed.), Genera et Species Plantarum Argentinarum II, pp. 1-273+CXX Lám. G. Kraft Edit., Buenos Aires.
- 1977. Asclepiadaceae. En T. Meyer, M. Villa Carenzo \& P. Legname (eds.), Flora Ilustrada de la Provincia de Tucumán, Primera Entrega, pp. 139271. Fundación Miguel Lillo, Tucumán.

MOORE, S. 1904. Asclepiadeae. En Mons. A. Robert's Matto Grosso plants II. J. Bot. 42 (496): 104.

SCHININI, A. 2005. Teodoro Rojas, biografía, viajes y especies dedicadas. Rojasiana 7: 101-149.

SIENRA ZAVALA, R. 2010. Síntesis de la Guerra del Chaco. Versión paraguaya - Versión boliviana. Concepción (Paraguay). 296 pp.

ULMKE, C. \& L. AUGUST. 2004. Kleiner Planzenfuhrer fur den Paraguayischen Chaco. Una guía para plantas nativas del Chaco paraguayo. Ed. INTAS, Loma Plata. 243 pp.

Original recibido el 16 de octubre de 2013; aceptado el 29 de noviembre de 2013. 